\title{
Selbsterfahrungen mit Kneipp-Anwendungen bei Wanderungen in den Schweizer Alpen
}

\author{
Jürgen Rohde \\ Chefarzt iR Helios-Klinikum Berlin-Buch, Deutschland
}

D ie randomisierte, kontrollierte Studie ist zwar wissenschaftlich gesehen die beste Methode, Therapien in ihrem Wert für den Patienten zu untersuchen [1]. Andererseits sind jedoch auch die klinische und die ärztliche Erfahrung von Bedeutung und sollten nicht vernachlässigt werden [2]. Die Erfahrungsheilkunde ist eine alte Wissenschaft, und es gab bereits Mitte des 19. Jahrhunderts die „Zeitschrift für Erfahrungsheilkunst“ [3]. Schon lange vor Pfarrer Sebastian KNeIPP (18211897) hat Vinzenz Priebnitz (17991851) Kaltwasseranwendungen und Abhärtungsmassnahmen (z.B. Barfusslaufen und „Taufussbäder") und andere Naturheilverfahren kurmässig von seinen Patienten in Gräfenberg durchführen lassen [4,5,6,7]. Prießnitz ist heute leider in Vergessenheit geraten, so dass man nur noch von den Kneipp'schen Kaltwasseranwendungen spricht [8]. Auch heute noch läuft die medizinische Praxis Gefahr, ohne klinische Erfahrung und Sachverstand von der externen Evidenz beherrscht zu werden [9].

\section{Methodik}

Bei mehreren Wanderungen im September 2005 und 2007 in den Schweizer Alpen, z.B. im Verzaska- und MaggiaTal in den Tessiner Alpen sowie von Amden aus in den Glarner Alpen (1416 m ü. NN), haben wir unsere Selbsterfahrungen mit Kaltwasseranwendungen protokolliert. Wir haben kalte Unterarmbäder, Gesichtsanschwemmungen und Wassertreten nach Kneipp im Fluss oder an Kneipp-

Die ärztliche Erfahrung hat neben der randomisierten, kontrollierten Studie eine enorme Bedeutung und sollte nicht vernachlässigt werden. Bei Wanderungen in den Schweizer Alpen haben wir mit kalten Unterarmbädern, Gesichtsanschwemmungen und dem Kneipp'schem Wassertreten (z.T. in Kneipp-Anlagen des Schweizer Kneippverbandes) sehr gute Erfahrungen gemacht. Als Fehlläufe wurden die hochgekrempelten Ärmel und Hosen mit möglichen Abschnürungen erkannt. Als Ergebnis teilen wir unsere Selbsterfahrungen mit den genannten drei Kaltanwendungen mit und diskutieren sie an Hand der zahlreichen Angaben in der älteren und jüngsten Literatur. Dabei werden das Hochgebirgsklima in den Alpen, die Höhenluft und die UV-Strahlung als weitere Faktoren bei Wanderungen in den Alpen berücksichtigt.

Schlüsselwörter: Kalte Unterarmbäder, Wassertreten, Wanderungen in den Schweizer Alpen, Kneipp-Anlagen, Selbsterfahrungen

\section{Personal Experience with Kneippism while Hiking in the Swiss Alps}

Although randomized controlled studies are now the preferred scientific approach, medical experience is of enormous value and should not be neglected. While hiking in the Swiss Alps, the author had very positive experiences with cold forearm baths, facial rinses and Kneipp water stepping (partly in spas of the Swiss Kneipp Association). There was one negative experience, however: rolling up trousers up to the thighs and the sleeves above the elbows may hamper the venous flow and cause a sensation of constriction. The author describes and comments on his personal experience with the above-mentioned three cold therapies in light of the abundant early as well as most recent literature. Additional factors at work when hiking in the Alps such as the alpine climate, the thin air and the UV rays were considered as well.

Key words: Cold forearm baths, water stepping, hiking in the Swiss Alps, Kneippism, personal experience

Anlagen durchgeführt. Der Schweizer Kneippverband hat 56 Kneipp-Anlagen, über die Schweiz verteilt, angelegt. Dabei handelt es sich z.B. um Kneipp-Wege (in Blitzingen), KneippGarten (in Grächen), Kneipp-BarfussWeg (in Mürren), Kneipp-Erlebnis-Pfad (in Langenbrück) u.a. Die Kneipp-Anlagen sind mit Anwendungstafeln beschildert, auf welchen die Grundregeln, Technik, Wirkung und Kontraindikationen der Armbäder und des Wassertretens genannt sind [10] (Abb. 1/2). Neben den Wirkungen des kalten Was- sers muss man das Hochgebirgsklima (Alpenluft und UV-Strahlung) berücksichtigen.

\section{Ergebnisse}

Folgende Selbsterfahrungen haben wir gemacht:

\section{Gesichtsanschwemmungen mit kaltem Wasser}

- Sie haben eine anregende und erfrischende Wirkung durch Verbes- 


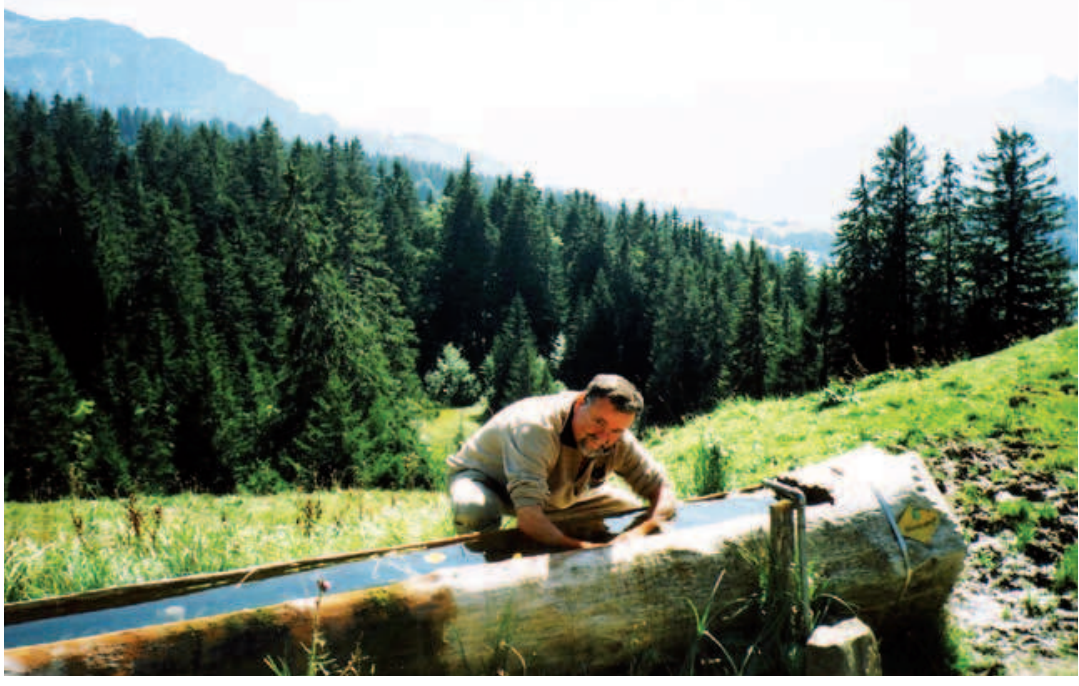

Abb. 1. Kalte Unterarmbäder oberhalb Amden, Glarner Alpen (1416 m).

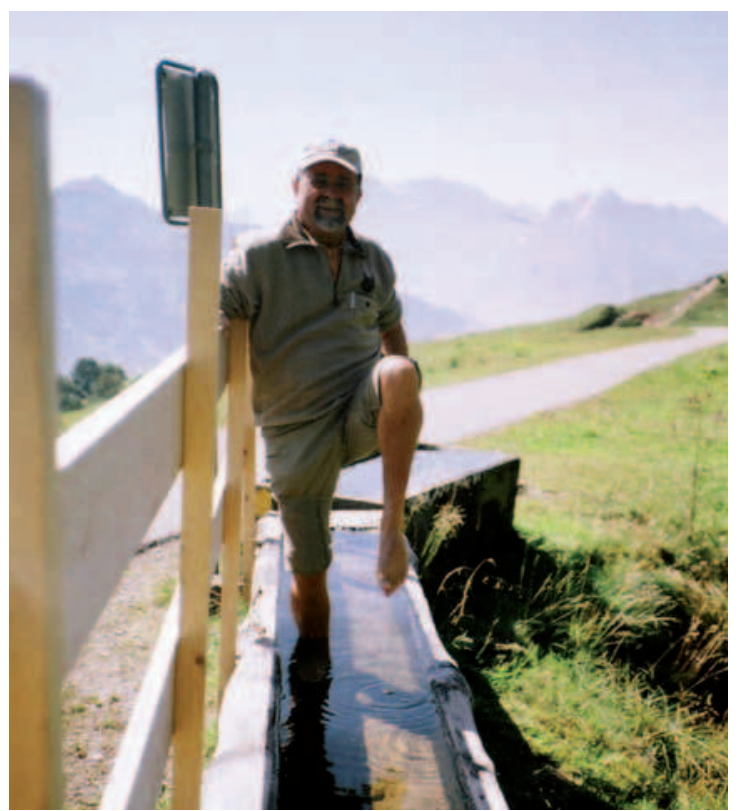

Abb. 2. Wassertreten, Kneipp-Anlage oberhalb Amden.

serung der Durchblutung der Gesichtshaut, die sich strafft. Deshalb wird der Gesichtsguss von den Kneipp-Anhängern auch als „Schönheitsguss“ bezeichnet.

- Man verspürt ein kribbelndes Gefühl im Gesicht und

- bei „müden Augen“ ein erfrischendes Gefühl.

- Man fühlt sich wacher („Weckeffekte“) und vigilanter.

- Bei den Kaltanwendungen ist ein tiefer Atemzug festzustellen und

- weiterhin ein Gefühl, als ob man nach der Ermüdung neue Kräfte schöpft.

\section{Kalte Unterarmbäder}

Sie sind etwas milder als der Armguss.

- Man spürt eine erfrischende Wirkung, besonders bei Hitzebelastung, körperlicher Überforderung und an warmen Sommertagen und

- eine anregende und belebende Wirkung, ohne dass sie aufregen (,Muntermacher").

- Sie beruhigen das Herz, als korrespondierendes inneres Organ.
- Sie verringern Schmerzen z.B. Epikondylalgie und Handgelenksschmerzen in Folge einer Arthrose.

- Die Bewegung der Arme im Wasser erzeugt dabei immer wieder einen neuen Kältereiz.

- Beim Leistungstief zwischen 11.00 und 15.00 Uhr haben sie eine sehr erfrischende Wirkung.

- Durch reaktive Erweiterung der Kapillaren in der zweiten Phase der Kältereaktion entsteht eine hellrote Verfärbung der Haut der gebadeten Unterarme mit gerader Begrenzung zur nicht gebadeten Haut des Oberarmes

- Bei Abgeschlagenheit, Müdigkeit und körperlicher Erschöpfung nach längerer Bergwanderung sind Unterarmbäder besonders erfrischend und prickelnd, wie Sekt.

- Entstandene Hitze im Kopf (mit Kopfschmerzen) wird abgeleitet.

- Man bekommt wieder Schwung, fühlt sich fit und wach, als ob Reserven eröffnet worden sind.

- Eine tiefe Atmung wird nach der Kaltanwendung angeregt und

- sie stimmt fröhlich, macht Appetit und wird als die „Tasse Kaffee“ der Naturheilkunde bezeichnet.

- Beim Menschen liegt die Hauttemperatur der oberen Körperhälfte mit Unterarmen und den Händen höher als die Hauttemperatur an der unteren Körperhälfte mit den Füssen. Deshalb erwärmt man sich nach einem kalten Unterarmbad schneller als nach dem Wassertreten. Die obere Hälfte des Körpers reguliert die Körpertemperatur des Menschen, nicht die untere mit den Füssen.

\section{Fehlläufe}

- Wenn man die Hemds- und Jackenärmel über das Ellenbogengelenk hinaus hochkrempelt, können venöse Abflussbehinderungen mit dem Gefühl des Abgeschnürtseins und der Kälte auftreten.

- Bei kalten Händen und Füssen können ein Gefühl der Kälte des ganzen Körpers und Schmerzen in den Unterarmen auftreten, das Unterarmbad ist dann sofort abzubrechen.

- Kaltreize wirken vormittags stärker als nachmittags. 


\section{Wassertreten}

- Es ist sehr erfrischend (morgendlicher „Muntermacher“), besonders im Sommer am Tage, und beruhigend abends.

- Es macht „müde“ Füsse wieder frisch, besonders nach Überforderung durch lange Fussmärsche.

- Wassertreten vitalisiert.

- Es fördert die Durchblutung. In der zweiten Phase der Kaltwasserwirkung auf die Kapillaren entsteht durch Kapillardilatation, wie bei den kalten Unterarmbädern, eine hellrote Färbung der Haut durch die Verbesserung der Durchblutung der Peripherie. Es entsteht ein prickelndes Gefühl.

- Der Kopf wird frei, depressive Verstimmungen verschwinden.

- Kopfschmerzen und Hitzegefühle im Kopf verschwinden.

- Wenn „heisse Füsse“ bei der Gebirgswanderung entstehen, so werden sie durch das Wassertreten beseitigt.

- Die Schweissfussbildung beim Wandern wird verringert und

- Muskelkater und Wadenkrampf (z.B. bei Varizen) beseitigt.

- Durch den „Storchengang“ beim Wassertreten werden schnell aufeinander folgende Kältereize erreicht.

- Bei Plantarflexion mit nach unten zeigender Zehenspitze und danach bei der Dorsalextension im oberen Sprunggelenk wird die sogenannte „Wadenmuskelpumpe“ betätigt, die den venösen Rückfluss anregt. Geschwollene Füsse schwellen ab.

- Nach dem Wassertreten fasst man neuen Mut und wandert fröhlich, hoffnungsvoll und erfrischt weiter.

- Wassertreten verringert die Schmerzen bei eigenem überfordertem Senk- und Spreizfuss und bei Sprunggelenksarthrose. Deshalb ist es günstig, nach dem Wassertreten die Füsse durchzukneten und auch Automobilisationshandgriffe durchzuführen [11].

- Bei beginnenden Kälteschmerzen sollte man das Wassertreten beenden, Füsse und besonders die Interdigital-Zwischenräume abtrocknen (sonst evtl. Gefahr der Pilzinfektion).

- Wassertreten dient bei serieller An-

Tab. 1. Selbsterfahrungen bei kalten Unterarmbädern, Gesichtsgüssen und Wassertreten, mit Wirkungen und Literaturangaben

\begin{tabular}{|c|c|}
\hline Selbsterfahrungen & Ursachen und Literaturangaben \\
\hline $\begin{array}{l}\text { Herz-Kreislauf- und Atemsystem } \\
\text { gerötete Haut und prickelndes Gefühl } \\
\text { tiefer Atemzug auf schroffe Kalt- } \\
\text { anwendung hin }\end{array}$ & $\begin{array}{l}\text { Verbesserung der kapillären Durchblutung } \\
\text { in der 2. Phase der Kaltreaktion [28] } \\
\text { zentrale Erregung des Vasomotorenzen- } \\
\text { trums und der Kreislauf-Atmungs-Steue- } \\
\text { rung [27] - Blutdruck und Pulserhöhung }\end{array}$ \\
\hline $\begin{array}{l}\text { ZNS } \\
\text { erfrischendes und belebendes Gefühl }\end{array}$ & vitalisierende Wirkung [2] \\
\hline $\begin{array}{l}\text { Verbesserung der Wachheit und } \\
\text { Aufmerksamkeit }\end{array}$ & $\begin{array}{l}\text { Vigilanztonus-Erhöhung, Verbesserung } \\
\text { zerebraler Durchblutung und Blutfluss- } \\
\text { Geschwindigkeit }[13,16]\end{array}$ \\
\hline Verbesserung der Stimmung & $\begin{array}{l}\text { euphorisierende Wirkung (Seebäder), er- } \\
\text { höhte Ausscheidung der 17-Ketosteroide [29] }\end{array}$ \\
\hline Schmerzlinderung & $\begin{array}{l}\text { Erhöhung der Schmerzschwelle [23], } \\
\text { Schmerzlinderung bei Kneipp-Kur [25], } \\
\text { Verringerung der Dysaesthesie und } \\
\text { Hypaesthesie bei Polyneuropathie [26] }\end{array}$ \\
\hline $\begin{array}{l}\text { Bewegungssystem } \\
\text { optimale Tonisierung der Muskulatur }\end{array}$ & kortiko-motorische-Kältereaktion $[15,16]$ \\
\hline Immunsystem & $\begin{array}{l}\text { Verbesserung der Reaktion des Immun } \\
\text { systems }[20,22] \text {, Anstieg der Schleimhaut- } \\
\text { temperatur durch kalte Fußbäder [30] um } \\
0,8^{\circ} \mathrm{C}\end{array}$ \\
\hline
\end{tabular}

wendung auch der sogenannten „Abhärtung“ und ist eine der populärsten Kneipp-Anwendungen mit den verbreitetsten Anlagen.

- Wassertreten kann man während einer Pause alle 2 Stunden durchführen. Es sollten keine häufigeren Pausen eingelegt werden, sonst ist der Laufrhythmus gefährdet. Es ist günstig, das Wassertreten mit einer Pause und einem Picknick zu verbinden, weil der Körperrhythmus, der durch die Kaltwasseranwendung ausgelöst wird, ca. 2 Stunden dauert.

\section{Fehlläufe}

- Bis an die Oberschenkel hochgekrempelte Hosen können zu venösen Abflussstörungen mit Abschnürgefühlen und kalten Füssen führen.

- Beim Wassertreten dürfen keine kalten Füsse bestehen, es entsteht sonst eine Zyanose und Frostgefühl in den Füssen.

- Man sollte nie nach dem Wassertre- ten ein kaltes Armbad nehmen, weil sonst der Körper auskühlt und man fröstelt. Deshalb sollten die Wassertret- und die Armbad-Anlage örtlich auseinander liegen. Beide Anwendungen stören sich gegenseitig.

- Wassertreten soll nicht bei arteriellen Durchblutungsstörungen der Beine und nicht bei Unterleibs-, Nieren- und Blasenerkrankungen durchgeführt werden.

\section{Diskussion}

Unsere Selbsterfahrungen mit den Kaltwasseranwendungen (Unterarmbad, Gesichtsanschwemmungen, Wassertreten) werden durch die Literatur gestützt, bestätigt und erklärt (Tab. 1). Im Folgenden sollen einige wichtige Literaturstellen diskutiert werden. Kalte Unterarmbäder (z.B. wie im Cold-Pressure-Test) verursachen eine Steigerung des systolischen und diastolischen Blutdrucks [12]. 
Kalte Gesichtsgüsse oder Anschwemmungen verbessern die mittlere zerebrale Blutfluss-Geschwindigkeit um 10\% [13] und die kognitive Hirnleistung im Alter [14].

Besonders die Kälterezeptoren des Gesichtes vermitteln dem Organismus thermoregulatorische Reaktionen. Das erkannte schon EBBEKE (1944) und nannte es „Trigeminusreflex“ oder „Wind- und Wetterreflex“ [15]. Er wies damit auf die Sonderstellung der Gesichtshaut für die Thermoregulation hin, weil der mittlere Trigeminusast besonders dicht mit Kälterezeptoren versorgt ist, wie man später fand. Schon beim Liegen im Freien oder am offenen Fenster verbessern sich deutlich Antrieb, Aufmerksamkeit und Stimmung [16]. Es ist oft eine euphorische Erregtheit, die man auch als „Luftrausch“ bezeichnet hat. Es ist eine Erhöhung des „Vigilanztonus“. Diese Änderung der Stimmung ist das psychische Äquivalent eines somatischen Vorganges und wird als „kortiko-motorische Kältereaktion“ bezeichnet. Durch die Kaltwasseranwendungen wird ein Rhythmus ausgelöst, der ca. 2 Stunden anhält [17].

Psychische Effekte der erfrischenden Wirkung des kalten Wassers sind seit langem bekannt. Diese „Sekundärwirkungen“ kommen von wärmeregulatorischen Zentren, und nach hydrotherapeutischen Kalt-Reizen kommt es zur Erhöhung des Muskeltonus (STERNBERG in [15]).

Auch V. Euler und SöDERBERg [15] fanden beim Absenken der Körpertemperatur oder beim Absenken der Temperatur des basalen Zwischenhirnes Veränderungen der elektrischen Aktivität des Neocortex und des Hypocampus (wie das im Wachsein, bei stärkerem Antrieb und Zunahme der Ymotorischen Aktivität typisch ist) mit dem Resultat der Erhöhung des Muskeltonus.

Scholz [ebenfalls 15] fand in psychologischen Tests nach kalten Teilanwendungen eine gesteigerte psychische Leistungsfähigkeit und Aufmerksamkeit. Es wird mit der o.g. kortikomotorischen Reaktion erklärt, und es wird von einem „positiven Gefühlsstau des Allgemeinbefindens“ gesprochen. Plötzliches Umschlagen des depressi- ven und gehemmten Verhaltens in eine erhöhte Aktivität und gesteigertes Selbstwertgefühl sind möglich. STIEFELHAGEN [18] vergleicht die Kaltwasserwirkung mit den Psychopharmaka.

Das Wassertreten führt zu einer Erhöhung des systolischen und diastolischen Blutdruckes um 20 bzw. 18 mm $\mathrm{Hg}$, und der Puls steigt um 20 Schläge an [19].

Nach Kneipp'schem Wassertreten kommt es zu messbaren Reaktionen des Immunsystems: Der Spiegel von Interleukin-6 wurde kräftig stimuliert; es wird als Gegenregulation des Immunsystems gedeutet und kann die sogenannte „Abhärtung“ nach Kneipp’schen Anwendungen erklären [20]. Die Abhärtung ist „ein Prozess, der zu einer verbesserten Abwehr gegen banale Infektionserkrankungen führt und mit einer geringen Infektionsrate sowie einem leichteren Krankheitsverlauf einhergeht“. Es ist eine Optimierung von Abwehr- und Bewältigungsleistungen gegen physische (Kaltreize) Stressoren [21]. Nach Kneipp'scher Hydrotherapie (z.B. Vollguss) treten signifikante Reaktionen des zellulären Immunsystems auf (spezifische Aktivierung von Helferzellen als spezifische Immunabwehr) [22].

Dass die Kältetherapie durch Hebung der Schmerzschwelle (um bis zu $75 \%)$ schmerzlindernd wirkt, ist bekannt und führte zur anerkannten postoperativen und posttraumatischen Kältetherapie [23,24]. LEUCHTGENS et al. [25] fanden nach einer 3-4-wöchigen Kneipp-Kur eine signifikante Besserung des Schmerzausmasses, mehrerer Aspekte der subjektiven Befindlichkeit und des Medikamentenverbrauches. Bei Polyneuropathien fanden UEHLEKE et al. [26] nach Kniegüssen und kalten Fussbädern eine signifikante mittlere Abnahme der Dysaesthesien und Besserung der Hypaesthesie. Nach Kneipp'schen Kaltwasserbehandlungen wurde in der Fernstudieeine Schmerzverringerung festgestellt. Der tiefe Atemzug auf eine schroffe Kaltanwendung hin bewirkt eine Erhöhung des Vasomotorentonus, vermehrte Kammerfüllung mit Pulsbeschleunigung und Blutdruckanstieg [27].

Weitere Faktoren bei Wanderungen in den Alpen sind neben dem kalten
Wasser auch das Hochgebirgsklima (Höhenluft, Sonnen- und UV-Strahlung) und die Wanderung selbst. Beim Hochgebirgsklima nehmen mit der Höhe die direkte UV-Strahlung, die Windgeschwindigkeit und die Andauer der Schneedecke zu, aber Temperatur, Partialdrucke von Sauerstoff und Wasserdampf nehmen ab. Weiterhin treten als Schonfaktoren im Hochgebirge eine fehlende Wärmebelastung und Allergenarmut (Reinheit) der Luft auf. Zur therapeutischen Nutzung eignen sich Höhenlagen zwischen 1000 und 2000 m über NN. Der komplexe Höhenreiz bewirkt eine Steigerung der Erythropoese mit vermehrtem Eiseneinbau in die Erythrozyten [31]. Aufgrund der Sauberkeit der Luft ist die UV-Bestrahlung durch die Sonne in der Höhe viel stärker als im Flachland. Daher sind die Abdeckung des Kopfes, der Augenschutz durch eine einwandfreie Sonnenbrille und Lichtschutzmittel für die den UV-Strahlen ausgesetzten Hautpartien unbedingt zu empfehlen. Die Alpen sind auch als Therapielandschaft bekannt. Seit Jahrhunderten haben die Menschen die Alpen aufgesucht, um dort gesund zu werden [32]. Die Alpen stehen für eine unverfälschte Natur (ohne Umweltverschmutzung) und Ursprünglichkeit. Sie stehen für eine „heile Welt“, was auch heute noch stimmt, wenn auch mit gewissen Einschränkungen.

Bei der Selbsterfahrung der Kaltwasseranwendungen bei Wanderungen in den Alpen spielen auch spezifisch alpine Faktoren eine grosse Rolle:

- Bei starker Sonnen- und UV-Bestrahlung sowie körperlicher- und Herz-Kreislaufbelastung, in Verbindung mit Schweissbildung, entsteht ein vermehrtes Bedürfnis nach $\mathrm{Ab}$ kühlung und Erfrischung sowie Durstgefühl.

- Aufgrund der trockenen Luft trocknen die Schleimhäute der oberen Atemwege eher aus und der Flüssigkeitsbedarf ist erhöht, man hat mehr Durst und trinkt mehr, d.h., man muss beim Wandern in den $\mathrm{Al}$ pen immer ein Getränk mitnehmen.

- Auf das Wohlbefinden haben positiven Einfluss: die Ruhe in den Bergen, der Blick ins Tal, die grünen 
Wiesen mit ihrem Duft (besonders das Heu, nach dem Mähen), die bunten Farben der Gebirgesflora und die saubere Luft.

- Negativen Einfluss auf die Psyche des Wanderers im Gebirge, nebst Angstgefühlen, haben schlechtes Wetter, Kälteeinbrüche und schlechte Sicht, wie z.B. bei Wolkenbrüchen oder starken Nebel mit Atembeklemmungsgefühlen und bei Schneestürmen mit Frostgefühlen. Das sind gleichzeitig auch die Gefahren bei Wanderungen in den Alpen, die man frühzeitig erkennen und auf die man schnell reagieren muss (Schutz suchen), weil sich das Wetter sehr abrupt ändern kann. Man muss die alpinen Faktoren kennen.

\section{Literatur}

1 Windeler J, Antes G, Behrens $\mathrm{J}$ et al. Kritische Evaluation ist ein Wesensmerkmal ärztlichen Handelns. Deutsches Ärzteblatt 2008;105(11):A 565-70.

2 Dobos G, Deuse U, Michalsen A: Chronische Erkrankungen integrativ. Elsevier, Urban und Fischer, München 2006.

3 Bernhardi A, Löffler F (Hrsg.): Zeitschrift für Erfahrungsheilkunst, III. Band, A. Hirschwald Verlag, Berlin 1849.

4 Priessnitz V: Vinzenz Priessnitz'sches Familien-Wasserbuch. Prießnitz' Diktat von 1847 (Fotokopie).

5 Rohde J: In Vergessenheit geratene Erkenntnisse über Prießnitz aus dem unbekannt gebliebenen Vinzenz Prießnitz'schen FamilienWasserbuch von 1847. Forsch. Komplementärmed. Klass. Naturheilkd. 2002;9:85-98.

6 Rohde J: Hungern und Diät nach dem Vinzenz Prießnitz'schen Familien-Wasserbuch von 1847. Forsch. Komplementärmed. 2007;17: 33-38.

7 Rohde J: Die Behandlung von Infektionskrankheiten nach dem Vinzenz Prießnitz'schen Familien-Wasserbuch von 1847 (Teil 1). Schweiz Zschr. GanzheitsMedizin 2008:20(4): 231-237. (Teil 2) ebenda, 2008;20(5):292-300.
8 Kneipp S: Meine Wasserkur. J. Kösel, Kemten (Bayern) 1886

9 Sackett D L, Richardson W S, Rosenberg W, Haynes R B: Evidenzbasierte Medizin. Zuckschwert, München 1996.

10 Schweizer Kneippverband: Vorstandshandbuch s. 8.1 und Dokumentation über Bau und Anwendung von Kneipp-Anlagen, 2007.

11 Rohde J: Automobilisation der Extremitätengelenke. Urban und Fischer, München, Jena 2003.

12 Klinker L, Walther E, Knape K: Einige Ergebnisse über die Bedeutung von Kaltluftliegekuren. Zschr. Physiother. 1984;36:405-413.

13 Doering T J: Hydrotherapeutische Güsse und Waschungen. Ärztezeitschrift für Naturheilkunde 1996;37:194-204.

14 Doering T J, Thiel J, Steuernagel B, Johannes B, Konitzer M, Niederstadt C, Schneider B, Fischer G C: Veränderungen kognitiver Hirnleistungen im Alter durch Kneipp-Anwendungen. Forsch. Komplementärmed. Klass. Naturheilkd. 2001;8:80-84.

15 Amelung W, Evers $\mathrm{H}$ : Handbuch der Bäderund Klimaheilkunde. Schattauer-Verlag, Stuttgart 1962

16 Schmidt-Kessen W: Klimatherapie des bekleideten Patienten. Zschr. für angewandte Bäder- und Klimaheilkunde 1965;12:225-263.

17 Krammer H: Sebastian Kneipp - Vorbeugen, lindern, heilen. Kneipp-Verlag Leoben, Wien 2005.

18 Stiefelhagen P: Kneipp'sche Güsse statt Psychopharmaka. MMW-Fortschr. Med. 147. Jg., 2005;18:4-6.

19 Bomski B, Franck O, Klinker L, Walther E: Über die Wirkung von kurzen Kaltreizen. Zschr. Physiother. 1986;38:165-169.

20 Werner GT, Dirnovac V, Penz MG: Immunologische Untersuchungen zur Wirkung der Kneipp'schen Hydrotherapie. MMW-Fortschr. Med. 140. Jg., 1998:566-569.

21 Pschyrembel W: Naturheilkunde und alternative Heilverfahren, 3. Auflage. de Gruyter-Verlag 2006, S. 1

22 Gruber R, Penz M, Bieger P. Immunologie der Abhärtungsreaktion nach Hydrotherapie ? Sofortreaktionen nach einmaligem Kaltreiz. Phys Rehab Kur Med 1996;6:72-79.

23 Schröder D, Anderson M: Kryo- und ThermoTherapie. G. Fischer, Stuttgart, Jena, New York 1995.

24 Kröling P, Schöps P, Mühlbauer M, Senn E et al.:Einfluss von Eis und Kaltluft auf die Schmerzschwelle im Bereich der Articulatio humeroradialis. Zschr. Phys. Med. Baln. Med. Klim. 1990;19:227.
25 Leuchtgens $\mathrm{H}$, Albus T, Uhlemann C, Vogler E, Pelka R B, Resch K L: Auswirkungen der Kneipp-Kur, einer standardisierten Komplex therapie, auf Schmerz, Lebensqualität und Medikamentenverbrauch: Kohortenstudie mit 1-Jahres-Follow-up. Forsch. Komplementärmed. 1999;6:206-211.

26 Uehleke B, Wöhling H, Stange R: A Prospective "Study by Correspondence" on the Effects of Kneipp Hydrotherapy in Patients with Complaints due to Peripheral Neuropathy. Schweiz. Zschr. GanzheitsMedizin 2008;20(5):287-291.

27 Kuhn H: Experimentelle Untersuchungen einiger hydrotherapeutischer Anwendungen nach Kneipp, Zschr. für angewandte Bäder- und Klimaheilkunde 1957;4:140-149.

28 Krauss H: Hydrotherapie. Verlag Volk und Gesundheit, Berlin 1990

29 Menger W, Dölp R: Über Beziehungen zwischen Euphorie an der Nordsee und erhöhte 17-Ketosteroidausscheidung. Zschr. für angewandte Bäder- und Klimaheilkunde 1968;15: 318-327

30 Pöllmann L: Temperaturänderungen der Schleimhaut des Mundes und des Rachens während kalter und wechselwarmer Fussbäder. Klin. Wochenschr. 1987;65:281-286.

31 Gutenbrunner C, Hildebrand G: Handbuch der Balneologie und medizinischen Klimatologie. Springer, Berlin, Heidelberg 1998.

32 Wyder M: Kräuter, Kröpfe, Höhenkuren. Die Alpen in der Medizin - Die Medizin der Alpen. Verlag Neue Zürcher Zeitung, Zürich 2003.

\section{Disclosure Statement}

The author declares that no financial or other conflict of interest exists in relation to the content of this article.

\section{Korrespondenzadresse}

Dr. med. Jürgen Rohde

Ibsenstrasse 80, DE-15831 Mahlow

Tel. ++49 (0) 3379-372915 